\title{
MEASUREMENT OF INTERFACE DAMAGE HETEROGENEITY
}

\author{
D. P. FIELD* and B. L. ADAMS** \\ * Alcoa Technical Center, Alcoa Center, PA \\ ** Brigham Young University, Provo, UT
}

\begin{abstract}
Both stereological and direct experimental procedures of estimating the distribution of interface normal orientations in a polycrystalline material are discussed in connection with the problem of intergranular damage heterogeneity. Two stereological procedures of obtaining the interface normal orientation distribution are compared. The mathematical analysis required in obtaining this distribution from stereological measures is briefly described. It is shown that the stereology incorporating the line length per unit area of interfaces, as seen on a test section, is superior to that which counts intersections per unit length of scan line. A discussion of the advantages and limitations of these techniques is included.
\end{abstract}

KEY WORDS Grain boundaries, intergranular damage, stereology.

\section{INTRODUCTION}

The growth and coalescence of voids on crystallite interfaces in polycrystalline materials is often life limiting for structural elements subjected to extreme conditions. It is almost always observed that the distribution of these voids is non-uniform. Interfaces normal to a given tensile stress axis are often, but not always, preferentially damaged. Damage on interfaces of similar orientation to a given stress axis is generally distributed heterogeneously, however, indicating that factors other than interface normal orientation play important roles in determining the damage resistance of a given boundary. Some of these local factors may include lattice misorientation, grain boundary energy, and chemical segregation at the interface, among others. A structural element made of material containing a high fraction of grain boundaries which are preferentially damaged under certain operating conditions may suffer premature failure. This observation motivates the development of microstructural distribution functions which incorporate descriptions of grain boundary structure.

An interface structure distribution function (ISDF) was developed by Adams and coworkers (1986, Zhao, et al. 1988, Morris et al. 1988). This function was utilized in estimating an interface damage function (IDF), which gives the area fraction of damaged crystallite interface, for oxygen-free electronic (OFE) copper crept in uniaxial tension (Adams, et al., 1990). Functional dependence upon interface normal orientation and crystallite lattice misorientation was incorporated into the analysis. In subsequent investigations, an additional parameter defining the plane of the interface in the crystallite lattice coordinate frame was considered (Field and Adams 1992a, 1992b). The lattice orientation of each 
crystallite with respect to an external coordinate frame is redundantly included among these parameters. Functional dependence upon alternate or additional parameters defining certain characteristics of the boundary, such as curvature or chemical composition, could be incorporated into the analysis as required.

The utility of these functions is recognized in that they can quantify the heterogeneity of interface damage. The character of grain boundaries on which damage most preferentially occurs can be explicitly described using this information. This detailed description of interfaces enables a more thorough investigation of microstructural mechanisms which contribute to void nucleation and growth. In addition, by knowing the character of boundaries which are readily damaged and should be avoided in a microstructure, the ISDF may be strategically altered to obtain a more damage resistant distribution of interfaces.

This paper discusses the analytical techniques available to estimate the distribution of damage as a function of grain boundary structure. The primary focus will be upon defining the experimental and stereological procedures required for the determination of IDFs, and isolating the techniques which are best suited to their measurement. The power of the techniques as well as their limitations are discussed.

\section{EXPERIMENTAL AND STEREOLOGICAL PROCEDURES FOR ESTIMATING THE DISTRIBUTION OF INTERGRANULAR DAMAGE}

In describing experimental and stereological techniques for determining IDFs, the focus will be upon defining the distribution of interface normal orientations. More explicitly, techniques for determining the function $S_{V}(\mathbf{n})$, which is the surface area of interface having normal orientation $\mathbf{n}$, per unit volume, will be described. $S_{V}(\mathbf{n})$ is more accurately defined as

$$
S_{V}=\frac{1}{2} \int_{\mathbf{n} \in S^{2}} S_{V}(\mathbf{n}) \mathrm{d} \mathbf{n}
$$

where $S_{V}$ is the stereological measure, surface area per unit volume, $\mathbf{n}$ is the interface normal orientation, and $S^{2}$ is the two-dimensional space covering the unit sphere. The factor of one-half preceding the integral is based on the assumption of non-sidedness, that is, $S_{V}(\mathbf{n})=S_{V}(-\mathbf{n})$. A discussion of the determination of lattice orientations and misorientations is beyond the scope of this paper, but there are a variety of techniques available which adequately measure crystallite lattice orientations.

\section{Direct Experimental Determination of $S_{V}(\mathbf{n})$}

The direct experimental measurement of $S_{V}(\mathbf{n})$ in polycrystals is possible by a number of procedures, each of which requires considerable experimental effort. Each method requires sectioning of the microstructure to reveal the lineal array of grain boundary intersections with the section plane. Three such methods are briefly discussed in the following paragraph. These are 1) serial sectioning through a polycrystalline material, 2) two-surface analyses, and 3) transmission electron microscopy (TEM). 
The first method considered is that of serial sectioning. A series of parallel plane sections, cut at increments much smaller than the grain size, through a single specimen retrieves $S_{V}(\mathbf{n})$ to the resolution of the spacing between planes. The thickness of each plane section increment must be accurately known for this method to be reliable. In addition, serial sectioning is extremely tedious in that the grain morphology of each section must be accurately determined. This requires the construction and analysis of a "grain map" for each section in retrieving $S_{V}(\mathbf{n})$. Unless grain morphology can be accurately estimated automatically through image analysis or some other technique, this method has limited utility in that statistically reliable information requires a large number of interface measurements. A second experimental method of determining $S_{V}(\mathbf{n})$ is to examine only the grain boundaries which are visible on two adjoining surfaces of the specimen. Two surface analyses directly retrieve the interface normal, $\mathbf{n}$, for a single grain boundary when the angle between the two surface normals is known (cf. Randle and Dingley 1989). When a sufficient number of these interface normal orientations are randomly measured, the distribution of grain boundary normal orientations is found and $S_{V}(\mathbf{n})$ may be reasonably estimated. A serious experimental drawback of this method is that the angle between the two surfaces must be accurately known, which is extremely difficult, or an uncertainty arises in the measurement of the interface normal orientation. A third experimental procedure of directly measuring $S_{V}(\mathbf{n})$ is through the use of transmission electron microscopy where the orientation of the grain boundary plane can be experimentally determined. The preparation of specimens for TEM investigations is tedious and prone to uncertainties since an accurate measurement of specimen thickness is necessary. In addition, grain boundaries can only be observed individually and a large number of these must be measured to retrieve the appropriate distribution of interface normal orientations. In fact, the accurate determination of $S_{V}(\mathbf{n})$ by any of the procedures mentioned above requires that a sufficient number of random measurements are made to ensure statistical reliability. For functions residing in five- or eight-dimensional orientation space, the number of interfaces which must be examined to achieve reasonable statistical reliability is well above the number which could be reasonably measured by any of the aforementioned methods.

\section{Comparison of Stereological Measures Used in Determining $S_{V}(\boldsymbol{n})$}

Two stereological measures which may be used in obtaining $S_{V}(\mathbf{n})$, and which are amenable to experimental determination, will be discussed in this section. Intersections per unit length of scan line in a direction $\mathbf{t}, P_{L}(\mathbf{t})$ and line length per unit area of a given section, $j$, with line normal lying at an angle $\omega$ to a given reference line, ${ }^{j} L_{A}(\omega)$, can each retrieve $S_{V}(\mathbf{n})$. The following paragraphs outline the fundamental equations and certain solutions as given in publications by Hilliard (1963) and Adams (1986). A comparison of the measures $P_{L}(t)$ and ${ }^{j} L_{A}(\omega)$ in obtaining $S_{V}(\mathbf{n})$ yields valuable insights into the experimental and mathematical difficulties of each of the procedures.

Formal derivations of the fundamental equations relating $P_{L}(\mathbf{t})$ and ${ }^{j} L_{A}(\omega)$ to $S_{V}(\mathbf{n})$ will not be made here. The reader is referred to Adams and Field (1991) for derivations of the fundamental equations and formulations of the numerical solutions in obtaining $S_{V}(\mathbf{n})$. It suffices here to present the integral relations 
between the measurable and desired functions:

$$
\begin{gathered}
P_{L}(\mathbf{t})=\frac{1}{2} \int_{\mathbf{n} \in S^{2}} S_{V}(\mathbf{n})|\mathbf{t} \cdot \mathbf{n}| \mathrm{d} \mathbf{n} \\
{ }^{j} L_{A}(\omega)=\frac{1}{2} \int_{0}^{\pi} S_{V}(v, \omega) \sin ^{2} v \mathrm{~d} v
\end{gathered}
$$

It should be noted that the coordinate frame in which $S_{V}(\mathbf{n})$, as given in Eq. (2), resides is that defined as the external or specimen coordinate frame. The $S_{V}(v, \omega)$ of Eq. (3) is defined in the jth section coordinate frame with $v$ and $\omega$ being the polar and azimuthal angles of the interface normal. $\omega$ is defined as the angle between a reference line in the test section plane and the normal to the line formed by the intersection of the grain boundary and the test section.

Each of the functions $P_{L}(\mathbf{t}),{ }^{j} L_{A}(\omega)$, and $S_{V}(\mathbf{n})$ can be expanded in Fourier series representations:

$$
\begin{gathered}
P_{L}(\mathbf{t})=\sum_{r=0}^{\infty} \sum_{t=-2 r}^{2 r} S_{2 r}^{\prime t} K_{2 r}^{t}(t) \\
{ }^{j} L_{A}(\omega)=\sum_{t=-\infty}^{\infty} L_{t}^{j} \mathrm{e}^{i t \omega} . \\
S_{V}(\mathbf{n})=\sum_{r=0}^{\infty} \sum_{t=-2 r}^{2 r} S_{2 r}^{t} K_{2 r}^{t}(\mathbf{n})
\end{gathered}
$$

where $K_{2 r}^{t}(\mathbf{n})$ are the surface spherical harmonic functions (cf. Bunge, 1982) and $S_{2 r}^{\prime t}$ and $L_{t}^{j}$ are constant coefficients determined experimentally. $S_{2 r}^{t}$ are also constant coefficients which define the desired function, $S_{V}(\mathbf{n})$. The solution of Eq. (2), similar to that given by Hilliard (1963), relates the coefficients $S_{2 r}^{\prime t}$ to $S_{2 r}^{t}$

$$
S_{2 r}^{t}=S_{2 r}^{\prime t} \frac{4 r+1}{2 \pi} \frac{1}{A_{2 r}}
$$

where $A_{2 r}$ are coefficients defining the absolute value of the cosine of an angle as expanded in a Fourier series of Legendre polynomials.

The solution for Eq. (3) was given by Adams (1986). The coefficients defining ${ }^{j} L_{A}(\omega)$ are related to those of $S_{V}(\mathbf{n})$ by the following equation

$$
L_{t}^{j}=\frac{1}{\sqrt{2 \pi}} \sum_{r=|t|}^{Q} \sum_{p=-r}^{r} S_{2 r}^{p} T_{2 r}^{t p}\left({ }^{j} g\right) I_{2 r}^{t}
$$

where $Q$ is the truncation order of the series expansion, $T_{2 r}^{t p}\left({ }^{j} g\right)$ are the generalized spherical harmonic functions with ${ }^{j} g$ defining a rotation from the test section coordinate frame to the reference coordinate frame. The $I_{2 r}^{t}$ are a series of constants defined by the following integral

$$
I_{2 r}^{t}=\int_{0}^{\pi} \bar{P}_{2 r}^{t}(\cos v) \sin ^{2} v \mathrm{~d} v
$$

Where $\bar{P}_{2 r}^{t}(\cos v)$ are the associated Legendre polynomial functions. Expression (8) is a linear set of equations, with complex values $L_{t}^{j}, S_{2 r}^{p}$, and $T_{2 r}^{t p}\left({ }^{j} g\right)\left(I_{2 r}^{t}\right.$ being 
real), that can be solved for the unknown coefficients defining the desired function, $S_{V}(\mathbf{n})$.

To compare the two stereological procedures a hypothetical microstructure, having interfaces of a single normal orientation, was formulated. The function $S_{V}(\mathbf{n})$ of the hypothetical microstructure becomes a delta function centered at $\mathbf{n}^{\circ}$, the orientation of the interface normal. More specifically,

$$
\frac{S_{V}\left(\mathbf{n}^{\circ}\right)}{S_{V}}=\int_{\mathbf{n} \in S^{2}} \delta\left(\mathbf{n}-\mathbf{n}^{\circ}\right) \mathrm{d} \mathbf{n}=1
$$

where $S_{V}$ is the total surface area per unit volume in the structure, and $\delta\left(\mathbf{n}-\mathbf{n}^{\circ}\right)$ represents the Dirac delta function. The coefficients defining the series expansion of $S_{V}(\mathbf{n})$ are

$$
{ }^{T} S_{2 r}^{t}=K_{2 r}^{* t}\left(\mathbf{n}^{\circ}\right)
$$

where the ${ }^{*}$ denotes the complex conjugate function. The pre-superscript, $T$, denotes coefficients defining the "true" function. This "true" function can now be compared with "measured" functions determined from stereological analysis and implementation of Eqs. (7) and (8).

The coefficients required in the Fourier series expansions of $P_{L}(t)$ and ${ }^{j} L_{A}(\omega)$ for this hypothetical microstructure can each be determined analytically. Scan directions and test section normal orientations are given, and the coefficients are determined assuming perfect statistical reliability. This takes all statistical error out of the analysis and the two methods are analyzed solely on their respective abilities to determine the desired coefficients. For the $P_{L}(t)$ measure the scan directions are based upon equal angle increments through the polar and azimuthal angles. The number of scan directions is varied through several trials. The coefficients of the function $S_{V}(\mathbf{n})$ are subsequently determined from Eq. (7). For the ${ }^{j} L_{A}(\omega)$ measure the test sections must be chosen for each set of coefficients determined. The Euler angles, which indicate the orientation of one coordinate frame relative to another (cf. Bunge, 1982), of the sections used in the following analysis are indicated in Tables 1 and 2. Equation (8) is then employed to determine the coefficients of the desired function.

A quantitative comparison of the "measured" and "true" functions is enabled by defining the following parameter $\xi$,

$$
\xi=\oint_{\mathbf{n} \in S^{2}}\left[{ }^{T} S_{V}(\mathbf{n})-{ }^{M} S_{V}(\mathbf{n})\right]^{2} \mathrm{~d} \mathbf{n} .
$$

The pre-superscripts $T$ and $M$, in Eq. (12) indicate the "true" and "measured" functions respectively. This equation reduces to

$$
\xi(2 r)=\sum_{t=-2 r}^{2 r}\left[{ }^{T} S_{2 r}^{t}-{ }^{M} S_{2 r}^{t}\right]^{2}
$$

when the Fourier series expansions are substituted into the formulation. $\xi$ is a function of $2 r$ which is the order of the coefficient considered.

In utilizing the measure ${ }^{j} L_{A}(\omega)$, the maximum order of coefficients which can be accurately determined is fixed by the number of unique test section orientations. The analytical expression relating the coefficients of ${ }^{j} L_{A}(\omega)$ to those of $S_{V}(\mathbf{n})$, Eq. (8), mandates that there be $Q+1$ unique section planes analyzed. 
Table 1 Euler Angles, $v$ and $\omega$ for the sections used in the ${ }^{j} L_{A}(\omega)$ analysis.

\begin{tabular}{lrrrrr}
\hline Section & \multicolumn{1}{c}{$\boldsymbol{\varphi} 1$} & \multicolumn{1}{l}{$\boldsymbol{\Phi}$} & \multicolumn{1}{l}{$\boldsymbol{v}$} & \multicolumn{1}{l}{$\boldsymbol{l}$} \\
\hline 1 & 0 & 0 & 0 & 54.74 & 90.00 \\
2 & 90 & 90 & 0 & 54.74 & 90.00 \\
3 & 0 & -90 & -90 & 54.74 & 90.00 \\
4 & 0 & -45 & 0 & 54.74 & 90.00 \\
5 & 0 & 45 & 0 & 90.00 & 54.74 \\
6 & 135 & 45 & 0 & 9.74 & 90.00 \\
7 & 45 & 45 & 0 & 65.91 & 26.57 \\
8 & 45 & 135 & 0 & 114.10 & 26.57 \\
9 & 135 & 135 & 0 & 80.26 & 90.00 \\
10 & 90 & 45 & 0 & 35.26 & 0.00 \\
11 & 20 & 90 & 0 & 110.19 & 37.96 \\
12 & 90 & 135 & 0 & 90.00 & 54.74 \\
13 & -20 & -90 & 0 & 42.27 & 59.13 \\
14 & 45 & 90 & 0 & 90.00 & 35.26 \\
15 & 135 & 90 & 0 & 35.26 & 90.00 \\
16 & -45 & -60 & 0 & 5.26 & 90.00 \\
17 & -20 & -30 & 0 & 29.54 & 45.58 \\
18 & 20 & 30 & 0 & 70.88 & 38.45 \\
19 & 45 & 60 & 0 & 73.22 & 31.48 \\
20 & 45 & 120 & 0 & 106.78 & 31.48 \\
21 & 20 & 150 & 0 & 132.26 & 0.79 \\
22 & -20 & -150 & 0 & 97.47 & 69.63 \\
23 & -45 & -120 & 0 & 65.26 & 90.00 \\
\hline & & & & &
\end{tabular}

This is because the number of equations available to solve for the unknowns is dependent upon the number of section planes. There are four times the value of $2 r$ plus two (for real and imaginary zeroth order coefficients) unknowns in these equations, and four equations obtained from each section plane. Therefore, $Q+1$ section planes yield $4 Q+4$ equations to solve for $4 Q+2$ unknowns. This overdetermined set of complex linear equations is easily solved using numerical techniques. In utilizing the measure $P_{L}(\mathbf{t})$, data from a single scan direction can mathematically determine coefficients of $S_{V}(\mathbf{n})$ to infinite order. These coefficients, however, would be highly erroneous in almost all circumstances. The order to which the coefficients may be reasonably estimated is a function of the number of unique scan directions as well as of the actual directions scanned. This can be demonstrated by examining the $S_{V}(\mathbf{n})$ functions determined from the stereological analysis and comparing them with those known analytically.

Table 2 Combinations of section planes used in the ${ }^{j} L_{A}(\omega)$ analysis.

\begin{tabular}{ll}
\hline Number of sections & Sections analyzed (see Table 1) \\
\hline 3 (i) & $2,4,5$ \\
3 (ii) & $1,2,3$ \\
5 (i) & $2,6,7,8,9$ \\
5 (ii) & $2,10,11,12,13$ \\
7 & $2,6,7,8,9,14,15$ \\
9 & $2,4,5,6,7,8,9,14,15$ \\
13 & $2,10,11,12,13,16-23$ \\
\hline
\end{tabular}


To perform this investigation a microstructure having interfaces of a single normal orientation was considered. $n^{\circ}$ was fixed at a [111] orientation, or 45 and 54.7 degree azimuthal and polar angles. A "true" function was calculated by utilizing Eq. (11) to determine the coefficients of the Fourier series defined in Eq. (6). This function is shown plotted on a stereographic projection to various truncation orders in Figure 1. Figure 2 shows the same function as determined from $P_{L}(t)$ information collected on nine unique section planes and employing Eq. (7) and subsequently (6) to get $S_{V}(\mathbf{n})$. This is equivalent to scanning in directions defined by every twenty degrees in both the polar and azimuthal angles. The function is reasonably well represented to sixth order as seen from
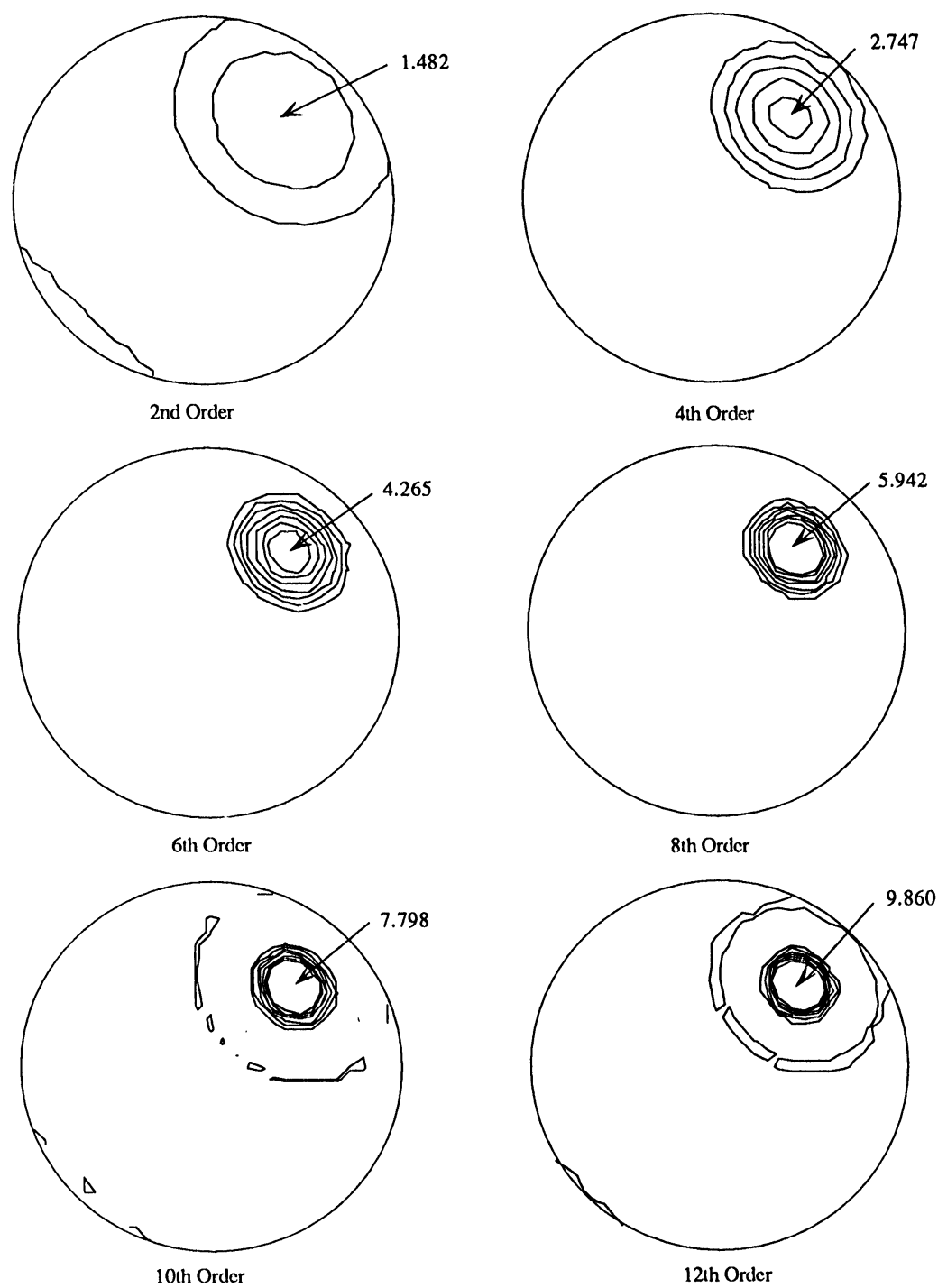

Figure 1 Stereographic plots of $S_{V}(\mathbf{n})$ as calculated from Eqs. (6) and (11), the "true" function. 

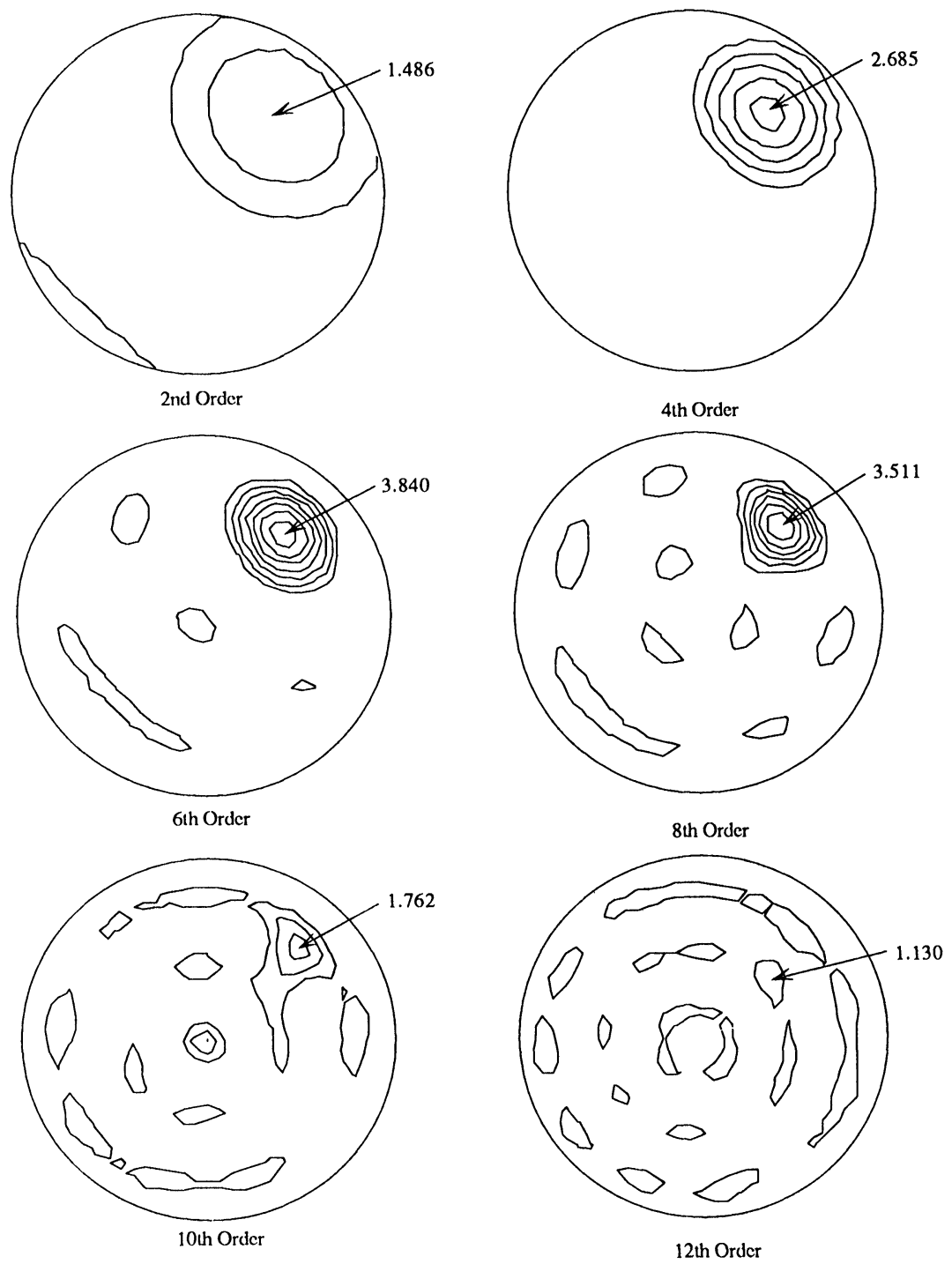

Figure 2 Stereographic plots of $S_{V}(\mathbf{n})$ as calculated from a nine section $P_{L}(\mathbf{t})$ measurement.

these plots. The magnitude of the peak shown on the plot of the eighth order truncation is lower than that shown for sixth order indicating that the eighth order coefficients were detrimental to the determination of the function. The higher order functions shown are, of course, nonsensical in that there were not enough scan directions considered to accurately obtain this information. That is to say, for this hypothetical microstructure, using $P_{L}(\mathbf{t})$ information, eighth order and higher coefficients cannot be determined without incorporating a higher number of strategically chosen scan directions into the analysis. For comparison, Figure 3 is given, which represents the same function as determined from ${ }^{j} L_{A}(\omega)$ 

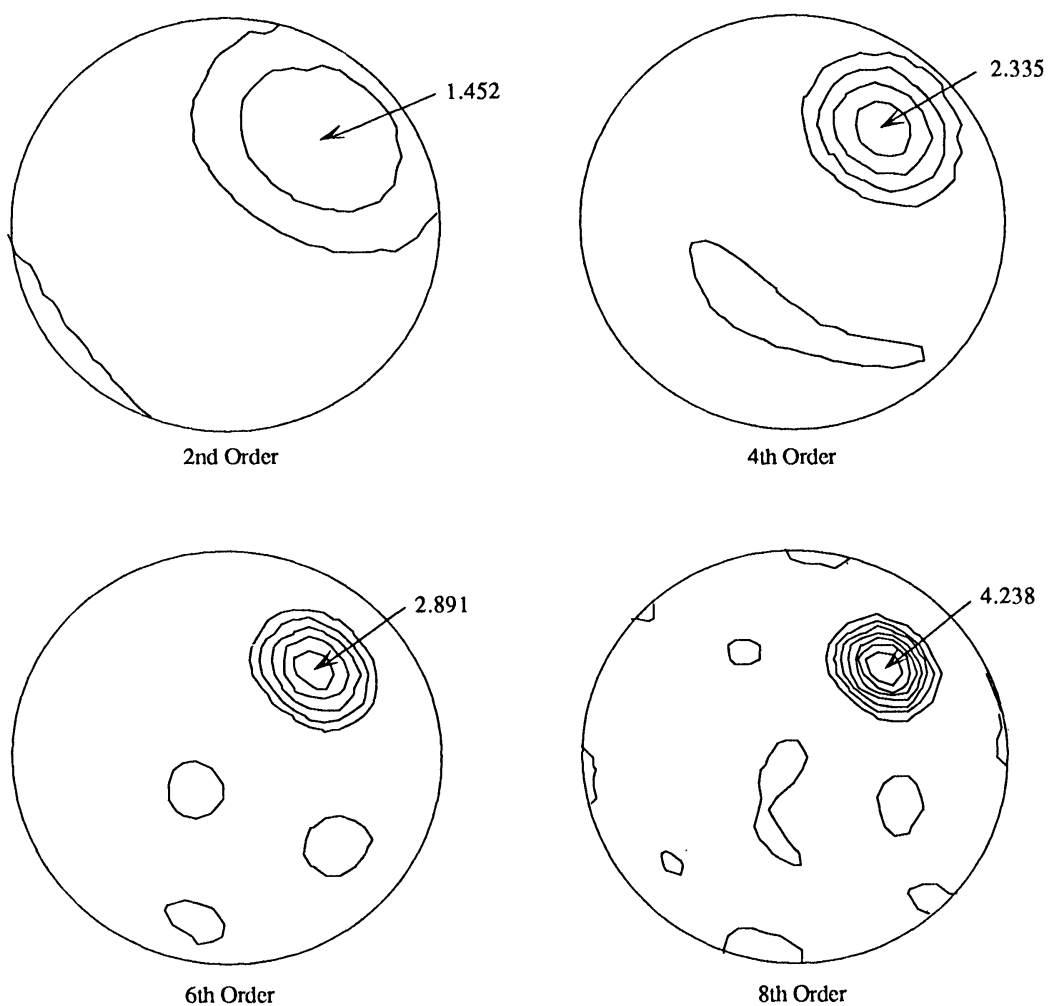

Figure 3 Stereographic plots of $S_{V}(\mathbf{n})$ as calculated from 3, 5, 7 and 9 section ${ }^{j} L_{A}(\omega)$ measurements.

information observed on three, five, seven, and nine section planes. Equation (8) is employed to give coefficients for the $S_{V}(\mathbf{n})$ function from the ${ }^{j} L_{A}(\omega)$ measure.

The parameter $\xi$, quantifying the accuracy of the stereologically measured functions, was determined for several different sets of scan lines or section planes for the two stereological measures considered. Figure 4 shows $\xi$ determined from use of the measure $P_{L}(t)$, as a function of truncation order. Figure 4(a) includes the complete set of information, and Figure 4(b) shows the same information on an expanded scale. Analysis of this information compared with the stereographic representations of the functions indicates that $\xi$ must be below 0.5 for a reasonable estimate of the true function. Figure 5 shows $\xi$ for $S_{V}(\mathbf{n})$ determined from the ${ }^{j} L_{A}(\omega)$ analysis using a variety of test section planes (defined in Tables 1 and 2).

Each of these stereological techniques has been utilized in the determination of IDFs and have yielded reasonable results (cf. Field and Adams 1992a, 1992b). However, a careful analysis of Figures 2-5 indicates that the ${ }^{j} L_{A}(\omega)$ stereology is superior to $P_{L}(\mathbf{t})$ in determining $S_{V}(\mathbf{n})$ for a delta type function, i.e. more reliable information can be obtained using fewer test section planes. This result can be generalized to include the determination of all $S_{V}(\mathbf{n})$ functions in that the general function can be thought of as a collection of delta functions. 


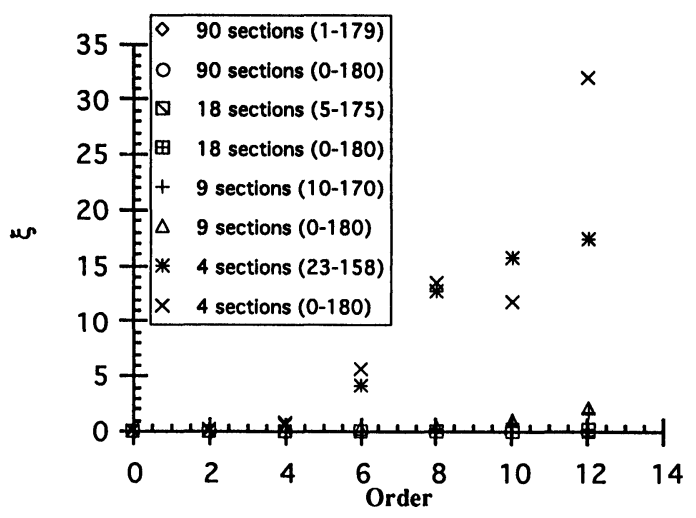

(a)

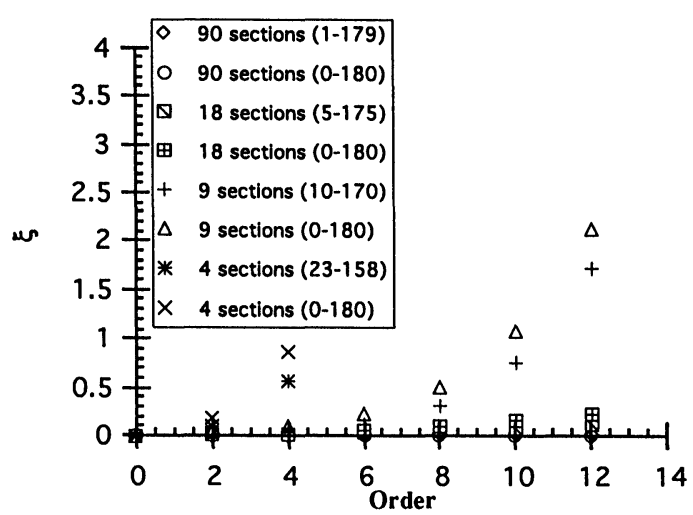

(b)

Figure 4 (a) The measure, $\xi$, plotted against truncation order for $P_{L}(\mathbf{t})$ analysis of $S_{V}(\mathbf{n})$. (b) An enlarged view of the bottom portion of (a).

\section{Incorporating Grain Boundary Features and Damage into the Analysis}

It has been demonstrated that stereological measures can be utilized to reliably determine the distribution of interface normal orientations in a polycrystal. The function $S_{V}(\mathbf{n})$, can be expanded to include other characteristics of grain boundaries, as suggested previously. This does not alter the logic of the stereological procedures described above. To incorporate additional information into the functions defined by the stereological measures, the domain of the given function is simply increased to include this information. If the new information is directly observable on a plane section, the same stereological procedures described above can be employed in the analysis. The Fourier series describing the function must be expanded to include the additional information desired, but the stereological solutions remain essentially unchanged. We define a new function, $S_{V}\left(\mathbf{n}, \phi_{1}, \phi_{2} \cdots\right)$, where the variables $\phi_{i}$ indicate various parameters describing the crystallite interface. These variables, as mentioned above, might include crystallite lattice orientations and misorientation, chemical composition, 


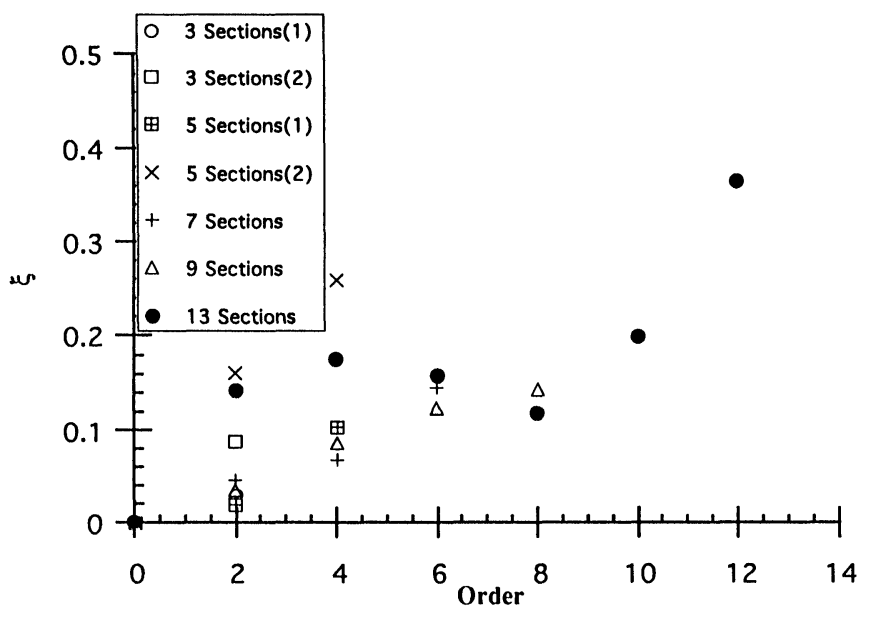

Figure $5 \xi$ plotted against truncation order for ${ }^{j} L_{A}(\omega)$ analysis of $S_{V}(\mathbf{n})$.

boundary curvature, etc. Each of the parameters used in describing the interface must be measurable, either directly or by statistical or stereological techniques, from plane surfaces.

Damage on the boundary is considered in a separate function of the same parameters and given by $S_{V}^{D}\left(\mathbf{n}, \phi_{1}, \phi_{2} \cdots\right)$, the superscript $D$ indicating damaged. This function is calculated from the damaged portion of the information obtained by the stereological measure. To determine an IDF, the area fraction of damage on boundaries of a given character, the quotient of the damaged and undamaged functions is taken

$$
S_{S}^{D}\left(\mathbf{n}, \phi_{1}, \phi_{2} \cdots\right)=\frac{S_{V}^{D}\left(\mathbf{n}, \phi_{1}, \phi_{2} \cdots\right)}{S_{V}\left(\mathbf{n}, \phi_{1}, \phi_{2} \cdots\right)} .
$$

$S_{S}^{D}$ indicates damaged surface area per unit surface, or area fraction of damaged crystallite interface.

The IDFs measured in crept OFE copper using this analysis have consistently indicated that certain special types of boundaries are preferentially damaged. The strength of this technique in measuring interface structure distributions and, more specifically, IDFs in isolating damage prone interfaces has been demonstrated in previous works (Field and Adams 1991a, 1991b). The reader is referred to these papers for a more complete discussion of the IDF determinations and discussions of the results.

\section{DISCUSSION}

There are several techniques available by which distributions of interface structure may be estimated. The stereological procedure of determining the distribution of interface normal irientations, presented here, is an important component of a powerful technique for estimating interface structure distribu- 
tions. A discussion of grain boundary structure using crystallite lattice orientation or misorientation distributions, including the coincident site lattice (CSL) theory, is incomplete without inclusion of information describing the plane of intersection of the two lattices. The difficulty in directly measuring interface normal orientations motivates the development of methods by which a large number of interfaces may be considered without directly measuring individual interface normal orientations.

The above discussion reveals both the strengths and the limitations of the stereological procedures for estimating interface normal orientation distributions. The stereological determination of the interface normal orientation distribution for the hypothetical microstructure, which contained interfaces of a single normal orientation, leads to the conclusion that stereological procedures are acceptable for this measurement. There are a number of important points to keep in mind, however, when utilizing these techniques. Firstly, the analysis performed in the previous sections assumes perfect statistical reliability in the test section plane. In performing actual measurements, it is important to analyze sections of the microstructure which are representative of the overall distribution. The sections must be large compared to the grain size so that edge affects of the region examined do not present a problem. In addition, there must be a sufficient number of boundaries analyzed on each test section to ensure accurate statistics (As the number of parameters which describe the distribution of interfaces increases (such as lattice misorientation, etc.) the number of boundaries which must be analyzed for statistical reliability increases exponentially.)

The selection of test section plane orientations on which interfaces are to be examined is another point which must be carefully considered. The number of unique section plane orientations required is fixed by the stereological procedure utilized as well as by the order at which the Fourier series expansion is to be truncated. The section normal orientations should then be chosen so as to uniformly cover the asymmetric region of the unit sphere. (In using the measure $P_{L}(\mathbf{t})$, the section orientations should be chosen so that the scan directions on the planes most uniformly cover the asymmetric region of the unit sphere).

This analysis does not lend itself well to the investigation of three grain junctions. These points may be extremely important, however, in determining the points in a polycrystal which are most preferentially damaged. To include this information in identifying the structure of damaged boundaries, the analysis must be expanded to incorporate stereological information on the orientation of each adjoining boundary. It must also include the lattice orientations of all grains which come in contact with a given interface, as seen in the section plane. An additional limitation of the method presents itself in considering those interfaces for which $S_{V}(\mathbf{n})$ is very small. The damaged portion of the function would be correspondingly small. Due to truncation errors of the series expansions, however, boundaries whereon $S_{V}(\mathbf{n})$ is very small could artificially indicate that they exhibit a high degree of damage. This problem arises because $S_{V}(\mathbf{n})$ is in the denominator of Eq. (14). The typical procedure is to disregard the boundaries for which $S_{V}(\mathbf{n})$ is less than 10 percent of the maximum value.

The obvious advantages of using stereological techniques to retrieve interface normal distributions lie in the ability to incorporate a large amount of information into the analysis with a reduced amount of experimental effort (as compared with TEM, serial sectioning, or two-surface analyses). As indicated by Eq. (14) the 
limit to the amount of information which may be included is the statistical reliability which can be achieved in analyzing a single section plane. The methods described enable a complete geometrical description of the local character of crystallite interfaces (excepting three grain junctions).

A large number of test sections is not required to achieve reasonable resolution for the distribution of interface normal orientations. As seen in Figures 1-3, a truncation order of six, or even four, yields an acceptable estimate of the interface normal orientation. If more exact information is desired to pinpoint structural characteristics of preferentially damaged interfaces, subsequent bicrystal or individual grain boundary studies could be performed. The IDF measurements would define the character of the bicrystal that should be studied to retrieve the optimum information.

\section{CONCLUSIONS}

While investigations of crystallite interface structure distributions by direct experimental measurement are possible, they are always tedious and prone to statistical uncertainties. In comparing the advantages and limitations of directly measuring the interface normal orientation to those of retrieving the distribution of normal orientations through stereological techniques, stereology proves to be superior in incorporating a large number of measurements into the analysis. Each of the procedures discussed of directly measuring the interface normal orientation has serious drawbacks. Coupling this with the consideration that a large number of these measurements must be made for reasonable statistical reliability, it becomes unrealistic and essentially impossible to use these techniques in estimating IDFs. While obtaining an accurate estimate of the distribution of interface normal orientations through stereological techniques requires a large amount of information, this same amount of information would be required for statistical reliability in directly measuring the normal orientation distributions. While the experimental effort for the stereological measurements is significant, it is orders of magnitude less than that required in directly measuring the normal orientations of a similar number of interfaces.

Stereological techniques for estimating distributions of interface structure and intergranular damage are powerful tools in studying the correlation between grain boundary structure and damage. Each of the procedures discussed in this paper, that of Hilliard (1963) utilizing $P_{L}(\mathbf{t})$, as well as that of Adams (1986) incorporating ${ }^{j} L_{A}(\omega)$, are amenable to the investigation of damage as a function of parameters describing the local interface character. The stereology of ${ }^{j} L_{A}(\omega)$ has been shown to be superior to that of $P_{L}(\mathbf{t})$ in estimating the function $S_{V}(\mathbf{n})$. The measure ${ }^{j} L_{A}(\omega)$ requires fewer section planes and achieves superior accuracy than $P_{L}(\mathbf{t})$ in estimating $S_{V}(\mathbf{n})$.

\section{Acknowledgement}

The authors wish to acknowledge support of this work by the Office of Basic Energy Sciences of the US Department of Energy. 


\section{References}

Adams, B. L. (1986). Metall. Trans. 17A, 2199.

Adams, B. L., Zhao, J. and O'Hara, D. (1990). Acta metall. mater., 38, 953.

Adams, B. L. and Field, D. P. (1992). Metall. Trans., 23A, 2501.

Bunge, H. J. (1982). Texture Analysis in Materials Science, Butterworths, London.

Field, D. P. and Adams, B. L. (1992a). Acta metall. mater., 40, 1145.

Field, D. P. and Adams, B. L. (1992b). Metall. Trans., 23A, 2515.

Hilliard, J. E. (1963). Trans. Am. Inst. Min. Engrs., 224, 1201.

Morris, P. R., Zhao, J. and Adams, B. L. (1988). Metall. Trans., 19A, 2611.

Randle, V. and Dingley, D. (1989). Scripta metall., 23, 1565.

Zhao, J., Koontz, J. S. and Adams, B. L. (1988). Metall. Trans. 19A, 1179. 